\title{
Multi-Sensor Data Fusion for Autonomous Vehicle Navigation and Localization through Precise Map
}

\author{
Tehrani Niknejad Hossein ${ }^{1)}$ Seiichi Mita ${ }^{2)}$ Long Han $^{3)}$ Quoc Huy Do ${ }^{4)}$ \\ 1).2).3) 4). Toyota Technological Institute, Joint Research Center for Smart Vehicles \\ 2-12-1 Hisakata, Tempaku-ku, 468-8511, Nagoya, Japan (E-mail: \{tehrani, smita, han, sd09601\}@toyota-ti.ac.jp)
}

Received on July 7, 2011

Presented at the JSAE Annual Congress on May 18, 2011

\begin{abstract}
Inexpensive implementation of localization and environment mapping are critical issues for urban autonomous driving. We present a practical and low-cost navigation architecture to fuse different data from vehicle onboard sensors and estimate the vehicle state when individual observations such as GPS are noisy. We are trying to compensate the GPS errors by data fusion from different sensors in a probabilistic way and a particle filter with joint observations model has been proposed. We have evaluated the feasibility of proposed localization and navigation architecture for fully autonomous driving by doing many experiments in our campus including up and down slopes.
\end{abstract}

KEY WORDS: (Standardized) Electronics and Control, Autonomous Driving System, Vehicle Sensor, (Free) Precise Map, Sensor Fusion [E1]

\section{Introduction}

In urban environment with narrow roads and highly dynamic conditions, we need accurate and precise estimation of autonomous vehicle state for real time navigation and control. Inaccurate vehicle state estimation can cause driving outside the road corridor or leading to poor navigation decisions. Nowadays, the challenge is to develop high-performance navigation system solutions using low-cost sensor technology ${ }^{(1)}$. Localization and defining the path for autonomous driving need a digital map of the urban environment as a higher level of knowledge for realistic and practical urban autonomous driving. There are some cheap commercially available digital maps of urban cities mostly based on the GPS data such as Google Earth. Generally, current commercially available in-car navigation systems match the information from a GPS receiver with a digital map, i.e., map matching ${ }^{(2)}$. Though in urban environments, buildings may partly block satellite signals, forcing the GPS receiver to work with a poor geometric constellation of satellites, thereby reducing the accuracy of the position estimates ${ }^{(3)}$. Even worse, less than four satellites may become available, making position fixes impossible and interrupting the continuity of the navigation solution. Moreover, multipath propagation of the radio signal due to reflection in surrounding objects may lead to decreased position accuracy without notification by the GPS receiver, thereby reducing the integrity of the navigation solution ${ }^{(4)}$.

By using dual-frequency receivers and carrier-phase measurements, such as the teams of DARPA challenge used in their competition for localization, it is possible to achieve realtime position accuracy on a decimeter level ${ }^{(5,6)}$. However, the required receiver units are currently far too costly for use in commercial in-car navigation systems. Even if the GNSS receivers' positioning accuracy is enhanced by various augmentation systems, the problems of poor satellite constellations, satellite signal blockages, and signal multipath propagation in urban environments still remain. Therefore, to counteract navigation solution degradation in situations with poor satellite constellation geometry, shadowing, and multipath propagation of satellite signals, advanced in-car navigation systems commonly use complementary navigation methods, relying upon information from sensors such as accelerometers, gyroscopes, odometers and lasers ${ }^{(7)}$. In urban cities of Japan, many accurate digital maps have already developed and are commercially available ${ }^{(8)}$. These digital maps can be used as a knowledge map which provides useful information such as driving path, route and landmarks for autonomous driving. In this paper, we propose a practical and low-cost navigation architecture for fully autonomous driving based on the data provided by the Google Earth maps including driving path and landmarks positions. To enhance the Google Earth map data and improve positioning precision and reliability, it is necessary to adopt some sensor fusion algorithms, which estimate vehicle state and compensate sensor errors (such as gyro, GPS data, odometer, etc).

We have adopted a particle filter with joint observations model to fuse localization observations from different sensors of the autonomous vehicle. We briefly discuss the available localization observations including GPS data, vehicle dynamic model, landmarks. We present an adaptive joint observations model to integrate all observations and try to optimize it using Markov Chain Monte Carlo (MCMC) method. The structure of this paper is as follows; Section 2 introduce the reference frames for navigation. Section 3 describes joint observations model; Section 4 discuss in detail about different localization observations; Section 5 presents multi observations particle filter for vehicle localization; Section 6 presents autonomous driving and navigation architecture, and finally we talk about vehicle platform, do case studies and analyze the results of experiments. 


\section{Reference Frames for Navigation}

When the vehicle travels through the environment, perceptual data from environment were distilled into two maps including local occupancy grid map and global map. We consider both local and global maps for different tasks in autonomous driving including path planning, obstacle avoidance, positioning, etc. The eight meters local grid stores the obstacle information including sizes and positions and is mainly used for path planning and obstacle avoidance tasks. The coordinate of the local grid map corresponds to the vehicle body coordination as shown in the Fig.1. The local map is generated and updated by processing data from laser scanners every 50 millisecond. The real time information about the obstacles are stored in the grid format which will be used by path planner and movement control module to find suitable paths and avoiding obstacles.

The global map is defined to represent the vehicle's global position. We use Google Earth maps as a global reference for mapping the urban environment and Google Earth ${ }^{(9)}$ software has been used to define the predefined path and way points for autonomous driving of vehicle as shown in the Fig.1. By considering a reference point, we transform latitude, longitude, and altitude (LLA) frame to the local tangent plane coordination system. This coordinate system has its origin coinciding to vehicle coordination, but its $\mathrm{x}$-axis always points towards geodetic north, the $\mathrm{z}$-axis towards the origin of the earth-frame, and the y-axis completes the right-handed orthogonal frame on the geodetic reference ellipse. We use WGS-84 standard to convert the LLA frame of Google Earth to Cartesian coordinates $\left(x^{g p s}, y^{g p s}\right)$ expressed in a local tangent frame. The vehicle's path and way points are stored in the global map. We use the $\left[C_{b}^{n}\right]$ and $\left[C_{n}^{b}\right]$ transformation matrixes for transforming from local to global coordination and vice versa ${ }^{(10)}$.

\section{Joint Observations Model}

Estimating vehicle state is a key prerequisite for precision autonomous driving. The position and state of vehicle at time $t$ is expressed by the following vector;

$$
X_{t}^{v}=\left(x_{t}, y_{t}, z_{t}, \psi_{t}, \varphi_{t}, \theta_{t}, \delta_{t}, s f_{t}\right)
$$

- $\left(x_{t}, y_{t}, z_{t}\right)$ : vehicle position at global map at time $t$

- $\psi_{t}, \varphi_{t}, \theta_{t}$ : yaw, roll and pitch of vehicle at time $t$

- $\delta_{t}$ : front tire angle at time $t$

- $s f_{t}$ : tire scaling factor at time $t$

A single noisy sensor or observation such as GPS signals are usually unable to provide full knowledge about the vehicle's position and it is necessary to fuse several observations $s_{t}^{k}(k=$ $1, \ldots . K$ ) provided by different sensors. Each observation is related to the hidden state of vehicle and by using observation probability law $p\left(s_{t}^{k} \mid X_{t}\right)$ of individual observation, we can write following formula corresponding to state evolution of vehicle with multiple observations.

$$
\begin{array}{ll}
X_{t}^{v} & =f_{t}\left(X_{t-1}^{v}, u_{t}, v_{t}\right) \\
s_{t}^{k} & =h_{t}^{k}\left(X_{t}^{v}, w_{t}^{k}\right) \quad \mathrm{k}=1, \ldots \mathrm{K}
\end{array}
$$

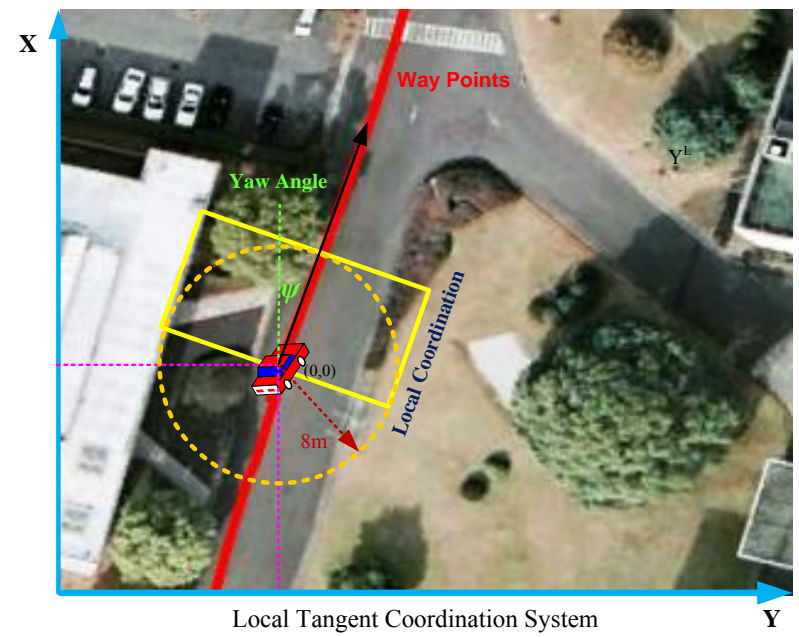

Fig. 1 Global and local coordination system

where $k$ is the number of the observations. $f_{t}$ is a (nonlinear) function, $v_{\mathrm{t}}$ is a white noise and $\mathrm{u}_{\mathrm{t}}$ is driving force. The time index $t$ is assumed to be an integer $t \in\{1,2,3, \ldots\}$. Equation (2) can be equivalently written in terms of the conditional $p d f$ $X_{t}^{v} \sim p\left(X_{t}^{v} \mid X_{t-1}^{v}\right)$, where $\sim$ means -statistically distributed according to." For each sensor $k$, there are possible observation models $s_{t}^{k}$ which $h_{t}^{k}$ is (nonlinear) observation function and $w_{t}^{k}$ observation noise of the observation model of sensor $k$ at time $t$.

Maximum Likelihood Estimation (MLE) method has already proposed for combining different observations and make a joint observation model as shown in equation (3) ${ }^{(11)}$.

$$
q\left(s_{t} \mid X_{t}^{v}\right)=\prod_{k=1}^{K} p\left(s_{t}^{k} \mid X_{t}^{v}\right)
$$

where $s_{t}$ denotes to the joint observation model of all observations $s_{t}^{k}, k=1, \ldots K$. Through our experiments, we found that some observations such as vehicle dynamic model or landmarks are more accurate and robust compared to GPS observations. Instead of considering same weights for different observations, we propose the Weighted Maximum Likelihood Estimation (WMLE) to define and make joint observation $p d f$ model as shown in (4).

$$
q\left(s_{t} \mid X_{t}^{v}\right)=\prod_{k=1}^{K} p\left(s_{t}^{k} \mid X_{t}^{v}\right)^{\alpha_{t}^{k}}, \sum_{k=1}^{K} \alpha_{t}^{k}=1
$$

where $\alpha_{t}^{k}$ represents the importance weight of the observation type $k$ at time $t$.

We define a Bayesian sequential model for making joint observation model as shown in Fig.2. We are trying to maximize the likelihood for joint observations model by optimizing the importance weights of the individual observations. In order to tune importance weights adaptively, we use MCMC method for updating the importance weights. We update the importance weight of the individual observations through the vehicle state evolution with a particle filter which will be discussed later. The importance weight at time $t$ is updated by conditioning it to the importance weight at time $t-1$, individual observations, $s_{t}^{k}, k=1, \ldots K$ and estimated state of the vehicle as shown in the following. 


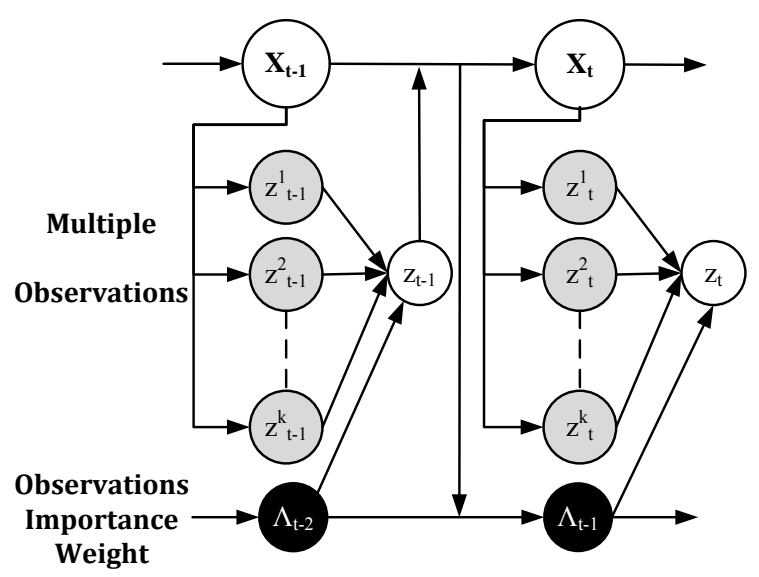

Fig. 2 Bayesian sequential model for making joint observation

$$
\begin{aligned}
& p\left(\Lambda_{t} \mid \Lambda_{t-1}, s_{t}^{1}, \ldots, s_{t}^{K}\right) \\
& =\int p\left(\Lambda_{t} \mid \Lambda_{t-1}, s_{t}^{1}, \ldots, s_{t}^{K}, X_{t}^{v}\right) p\left(X_{t} \mid \Lambda_{t-1}, s_{t}^{1}, \ldots, s_{t}^{K}\right) d X_{t}^{v}
\end{aligned}
$$

where $\Lambda_{t}=\left(\alpha_{t}^{1}, \ldots . \alpha_{t}^{K}\right)$. We use a particle filter for updating the vehicle state and $p\left(X_{t}^{v} \mid \Lambda_{t-1}, s_{t}^{1}, \ldots, s_{t}^{K}\right)$ corresponds to the weights of the particles. When the state of the vehicle is already known, we are able to estimate $p\left(\Lambda_{t} \mid \Lambda_{t-1}, s_{t}^{1}, \ldots, s_{t}^{K}, X_{t}^{v}\right)$. We use the following formula to estimate this likelihood.

$$
\Lambda_{t}=\lambda \cdot \Lambda_{t-1}+(1-\lambda) \cdot d\left(s_{t}^{1}, \ldots, s_{t}^{K}, X_{t}^{v}\right)
$$

which $d$ is a distance of the individual observation $s_{t}^{k}$ to the estimated state $X_{t}^{v}$

\section{Multi Observations}

In this section, we briefly discuss different observations corresponding to the autonomous vehicle localization. We consider three different observations $(K=3)$ for vehicle localization including vehicle dynamic model, landmarks and GPS data observations.

\subsection{Vehicle dynamic model $(k=1)$}

The data from IMU, odometer and steering angle have been fused in a Kalman filter to estimate the vehicle three dimensional movement in the global map.

The detail of the corresponding kalman filter has been presented in the previous paper ${ }^{(12)}$. In this paper, we have extended the vehicle dynamic model to consider three dimensional state of the vehicle in up and down slopes.

\subsection{Landmark detection and tracking $(k=2)$}

To limit the error of the positioning for long traveling distance in outdoor environment, we consider some landmarks with predefined positions and detect them by using laser scanner. The landmarks are detected by laser scanner in the local grid map according to their features and locations. In this paper, we define the construction cones as the land marks which are located along the experiment route. We have a set of $m$ landmarks $\Gamma=\left\{\left\{l_{i} \mid i=1, \ldots, m\right\}\right\}$ in the map which have been sorted in the $\Gamma$ according to their order of visiting by the autonomous vehicle. Each land mark $l_{i}$ has its characteristics or features as the following vector.

$$
l_{i}=\left(i, m_{i, x}, m_{i, y}, r_{i}\right)
$$

- $\quad i$ : index of the landmark in $\Gamma$.

- $\left(m_{i, x}, m_{i, y}\right)$ : position of the landmark $l_{i}$ in the global coordination system.

- $r_{i}$ : radius of the construction cone corresponding to the land mark.

We use the 2-D laser range scanner LMS291, produced by SICK. LMS291 has a maximum range of 80 meters, $180^{\circ}$ wide angle and the resolution is $0.5^{\circ}$. The laser sensor generates precise information about the objects around the vehicle and maps them into the rectangular coordinate corresponds to the vehicle body coordination system. The algorithm for land mark detection is described here which extracts the predefined features from laser scan data points and also estimates the positions of the objects in the global coordination system according to the current position of the vehicle. We just process the laser point's data which are located at the local map ( 8 meters $\times 8$ meters) for increasing the speed and efficiency of the landmark detection algorithm.

\section{Step 1: Clustering laser points}

We use Radial Bounded Nearest Neighbor (RBNN) graph to cluster the laser points into disjoint clusters ${ }^{(13)}$. Given $\mathrm{n}$ points in two dimensional space, at timestamp $\mathrm{t}, X^{t}=\left(p_{1}, p_{2}, \ldots p_{n}\right), p_{i}=$ $\left(x_{i}, y_{i}\right)$. We are going to make $\mathrm{m}$ disjoint clusters $C_{s}, s=$ $1, \ldots, m, C_{s} \cap C_{t}=\emptyset, \forall s \neq t$ such that every cluster contains at least one point. We use a Euclidean distance metric for calculating the distance between points. In this graph every node is connected to all neighbors that lie within a predefined radius $r$.We consider clusters with less than $n_{\min }$ points as noise or outliner data. In this paper, we consider $n_{\min }=2$.

The radius $r$ is determined according to laser point's density at local map and the landmark size. We have adjusted different values for $r$ by doing some experiments for different distances. We fit a rectangle $R E C_{s}=\left(x_{s}^{l}, y_{s}^{l}\right)$ to cluster $C_{s}$ which $\left(x_{s}^{l}, y_{s}^{l}\right)$ shows the center point of corresponding rectangle in the local coordination system.

\section{Step 2- Feature detection}

We define some features for every cluster and calculate the likelihood of each cluster corresponding to landmark $l_{i}$. We define a feature $f_{k}$ as a function $f_{k}: C \rightarrow \mathfrak{N}$ that takes a cluster $C$ as an argument and return a real value. Here, $C$ is the set of all possible clusters $\mathrm{C}_{\mathrm{s}}$, which we calculate the following four features;

1) Number of points in cluster $C_{s}, n_{s}=\left|C_{s}\right|$.

2) Circularity of cluster $C_{s}:$ As the construction cone has circular data points pattern in the laser scanner map, this feature measures the circularity of a cluster $C_{s}$. We sum up the squared residuals to a fitted circle and define the $x_{c}$, $y_{c}$ and $r_{c}$ as the circle center and radius. We can find the best fitted value by using the following formula;

$$
x_{s}=\left(A^{T} A\right)^{-1} A^{T} b
$$

where $x_{s}=\left(x_{c_{s}}, y_{c_{s}}, x_{c_{s}}^{2}+y_{c_{s}}^{2}-r_{c_{s}}^{2}\right)^{T}$ and

$$
A=\left[\begin{array}{ccc}
-2 x_{1} & -2 y_{1} & 1 \\
-2 x_{2} & -2 y_{2} & 1 \\
\vdots & \vdots & \vdots \\
-2 x_{n_{s}} & 2 y_{n_{s}} & 1
\end{array}\right], b=\left[\begin{array}{c}
-x_{1}^{2}-y_{1}^{2} \\
-x_{2}^{2}-y_{2}^{2} \\
\vdots \\
-x_{n_{s}}^{2}-y_{n_{s}}^{2}
\end{array}\right]
$$




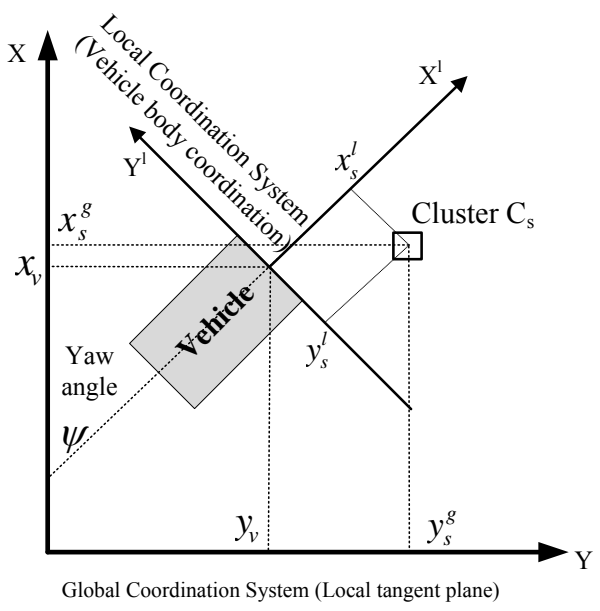

Fig. 3 Landmark position in global coordination system

$$
\left(x_{1}, y_{1}\right), \ldots\left(x_{n_{s}}, x_{n_{s}}\right) \in C_{s}
$$

The residual sum square is then calculated by (10)

$$
s_{c_{s}}=\sum_{i=1}^{n_{s}}\left(r_{c_{s}}-\sqrt{\left(x_{c_{s}}-x_{i_{s}}\right)^{2}+\left(y_{c_{s}}-y_{i_{s}}\right)^{2}}\right)
$$

3) Radius correspond to cluster $C_{s}$ : This feature calculate the radius of circle fitted to the cluster $C_{S}$.

4) Cluster position in global coordination system: If $\left(x_{v}, y_{v}\right)$ denotes to the current position of autonomous vehicle in the global coordination system and $\left(x_{s}^{l}, y_{s}^{l}\right)$ shows the center point of rectangle corresponding to cluster $C_{s}$ in the local coordination system, we are able to calculate the center point of rectangle in the global coordination system $\left(x_{s}^{g}, y_{s}^{g}\right)$ as shown in the Fig.3.

$$
\left[\begin{array}{l}
x_{s}^{g} \\
y_{s}^{g}
\end{array}\right]=\left[\begin{array}{l}
x_{v} \\
y_{v}
\end{array}\right]+\left[C_{b}^{n}\right]\left[\begin{array}{l}
x_{s}^{l} \\
y_{s}^{l}
\end{array}\right]
$$

\section{Step 3-Calculate the likelihood of clusters}

In this step, we calculate the likelihood of cluster $C_{s}$ corresponding to the landmark $l_{i}$ according to the features defined in the step 2 . We use a multivariate normal distribution to calculate the likelihood. The state variable $x_{s}^{i}$ includes 4 parameters which are calculated for each cluster $\mathrm{C}_{\mathrm{s}}$ corresponding to the landmark $l_{i} \in \Gamma$,

$$
x_{s}^{i}=\left(n_{s}, s_{c_{s}}, r_{c_{s}}-r_{i}, \sqrt{\left(m_{i, x}-x_{s}^{g}\right)^{2}+\left(m_{i, y}-y_{s}^{g}\right)^{2}}\right)
$$

The likelihood of cluster $C_{s}$ corresponding to features $f_{k}$ of $l_{i} \in \Gamma$ is calculated from the multivariate normal distribution as the following;

$p\left(C_{s} \mid f_{k} \in l_{i}\right)=\operatorname{det}(2 \pi \Sigma)^{-\frac{1}{2}} \exp \left(-\frac{1}{2}\left(x_{s}^{i}-\mu\right)^{T} \Sigma^{-1}\left(x_{s}^{i}-\mu\right)\right)(13)$

We use the maximum likelihood estimation (MLE) method for estimating $\mu_{k}, \Sigma_{k \times k},(k=1, \ldots 4)$. We have done some experiments for different positions and direction with construction cone to generate $\mathrm{n}$ samples for state variable of clusters corresponding to the landmark.

We calculate likelihood of cluster $C_{s}$ corresponding to $l_{i}$, $p\left(C_{s} \mid l_{i}\right)$ for all $l_{i} \in \Gamma$ at each scan, if there are some objects in the local grid map. We consider a threshold $\gamma$ for landmark likelihood. After detecting landmark, the vehicle position $\left(x_{v}, y_{v}\right)$ has been calculated according to position of the landmark $l_{k}$ by the following formula.

$$
\left[\begin{array}{l}
x_{v} \\
y_{v}
\end{array}\right]=\left[\begin{array}{l}
m_{k, x} \\
m_{k, y}
\end{array}\right]+\left[C_{b}^{n}\right]\left[\begin{array}{l}
x_{k}^{l} \\
y_{k}^{l}
\end{array}\right]
$$

After detection, we remove the landmark $l_{k}$ from $\Gamma$.

\subsection{GPS observation $(k=3)$}

In our experiments, we use low-cost GPS receiver, which has enormous errors. In order to reject the erroneous fixes caused by multi-path and satellite constellation changes, we use the following function to reject jumps.

$$
\text { reject }=\left|p_{t+1}^{g p s}-p_{t}^{g p s}\right|>\Delta X^{v}(1+\delta)+\varepsilon
$$

We can group the error sources of GPS receiver data into two classes; discontinuous errors( such as jumps) and continuous errors (drift and model errors). With every new state measurement, the change in position $\left|p_{t+1}^{g p s}-p_{t}^{g p s}\right|$ is checked for validity based on the measured $\Delta X^{v}$, from vehicle dynamic model with error $\delta$ and position jitter $\varepsilon$. In the above equation, we ensure that the reported motion of the vehicle by GPS is not significantly greater than the anticipated motion given the vehicle dynamic model.

\section{Particle Filter with Multiple Observations}

We apply the kalman filter for estimating yaw, roll, pitch and front tire angle of the vehicle. As our main contribution is to make adaptive joint observation model for estimating the vehicle position, we do not discuss the kalman filter which has been applied for estimation of mentioned parameters and mainly focus on estimating the vehicle position. We adopt and extend particle filter to fuse data from different observations and address the problem of position estimation of vehicle at time $t$. Here, the first state of the vehicle is known, and localization seeks to correct and compensate incremental and cumulative errors in vehicle control.

Different from the standard particle filter framework, we assume that there are multiple observations for the hidden state of the vehicle. A multi sensor switching particle filter has already proposed by switching observation and making joint observation $p d f$ by using MLE though, it considers same importance weight for individual observations ${ }^{(11)}$. We consider importance weight for different observations and propose WMLE for making joint observation $p d f$.

The goal of particle filter is to estimate a posterior probability density over the state space conditioned on the data collected so far. The data typically consists of an alternating sequence of time indexed observations $s_{t}^{k}, k=1, \ldots K$ which comes from the $K$ available sensors such as GPS, landmarks or the laser scanner and control measurement $u_{t}$ from control including odometer/IMU data and steering wheel which describe the dynamic of the vehicle. Each sensors or type of observations $k=1, \ldots K$ has specific accuracy and reliability which we present by $\alpha_{t}^{k}$ as the importance weight of observation type $k$ at time $t . \alpha_{t}^{k}$ are tuned adaptively for individual observations by using the MCMC method to maximize the likelihood for joint observation $p d f$.

If $\operatorname{Bel}\left(X_{t}^{v}\right)$ denote the posterior at time $t$ under the Markov assumption, the posterior can be computed efficiently by recursively updating the belief whenever new information is 
received. The multi observation particle filter has been presented as the following steps;

\section{Inputs:}

$\overline{V_{t-1}}=\left\{\Lambda_{t-1},\left\langle X_{t-1}^{v[m]}=\left(x_{t-1}^{[m]}, y_{t-1}^{[m]}, z_{t-1}^{[m]}\right), \omega_{t-1}^{m}\right\rangle \mid m=1, \ldots, M\right\}$ represent the $\operatorname{Bel}\left(X_{t-1}^{v}\right), \Lambda_{t-1}=\left(\alpha_{t-1}^{1}, \ldots, \alpha_{t-1}^{K}\right)$ the observations importance weight .

- Control measurement $u_{t}$ of the vehicle

- Individual observations $s_{t}^{k},(k=1, \ldots K)$. In this paper, we consider $K=3$ includes vehicle dynamic model, landmarks and GPS data observations.

\section{Particle Filter Loop}

For $m=1, \ldots, M$.

- Sample from the vehicle dynamic model and generate new prediction for vehicle state.

$$
\hat{X}_{t}^{v[m]} \approx p\left(X_{t}^{v} \mid u_{t}, X_{t-1}^{v[m]}\right), m=1, \ldots, M
$$

- Calculate the likelihood of generated samples through the following equation.

$$
\omega_{t}^{[m]}=\omega_{t-1}^{[m]} \cdot p\left(s_{t} \mid \hat{X}_{t}^{v[m]}\right), m=1, \ldots, M
$$

$p\left(s_{t} \mid \hat{X}_{t}^{v[m]}\right)$ is a joint model of all observations $s_{t}^{k}$. We use the weighted likelihood estimator and the weight of observation from previous step $\Lambda_{t-1}=\left(\alpha_{t-1}^{1}, \ldots . \alpha_{t-1}^{K}\right)$ to estimate the joint observation model at time $t$.

$\log \left(p\left(s_{t} \mid \hat{X}_{t}^{v[m]}\right)\right)=\alpha_{t-1}^{k} \cdot \sum_{k=1}^{K} \log \left(p\left(s_{t}^{k} \mid \hat{X}_{t}^{v[m]}\right)\right)$,

We can easily calculate the $p\left(s_{t}^{k} \mid \hat{X}_{t}^{v[m]}\right)$ by using a $p d f$ (for example normal distribution) corresponding to distance of observation $s_{t}^{k}$ from the sample $\hat{X}_{t}^{v[m]}$.

$$
p\left(s_{t}^{k} \mid \hat{X}_{t}^{v[m]}\right) \approx p d f\left(d\left(s_{t}^{k}, \hat{X}_{t}^{v[m]}\right)\right)
$$

sum $=\operatorname{sum}+\omega_{t}^{[m]}$ : Update normalization factor

$$
\text { endfor }
$$

\section{Update Importance Weight of Observations}

For $k=1, \ldots, K$

- Update the importance weight of the observations for next step of particle filter. $\Lambda_{t}=\left(\alpha_{t}^{1}, \ldots . \alpha_{t}^{K}\right)$ is updated through the following formula.

$$
\alpha_{t}^{k}=\lambda \cdot \alpha_{t-1}^{k}+(1-\lambda) \sum_{i=1}^{m} \omega_{t}^{[m]} \cdot d\left(s_{t}^{K}, X_{t}^{[m]}\right)(20)
$$

endfor

\section{Resampling}

- compute

$$
N_{e f f}=\left[\sum_{i=1}^{M}\left(\omega_{t}^{i}\right)^{2}\right]^{-1}
$$

if the $N_{\text {eff }}>\zeta$ then resample the particles

$\underline{\text { Return }} V_{t}=\left\{\Lambda_{t},\left\langle X_{t}^{v[m]}, \omega_{t}^{m}\right\rangle \mid m=1, \ldots, M\right\}$

\subsection{Sampling method}

We use the following algorithm for sampling from the distribution $p\left(X_{t}^{v} \mid u_{t}, X_{t-1}^{v}\right)$. The driving force is measured from odometer/IMU and steering potentiometer sensors from $\mathrm{t}$ until $\mathrm{t}+\Delta \mathrm{t}$ in the form $u_{t}=\left(x_{o d_{o}}, v_{\psi}, v_{\varphi}, v_{\theta}, v_{\delta}\right)$, where;

- $x_{o d o}:$ traveled distance from $t$ until $t+\Delta t$ by odometer,

- $\left(v_{\psi}, v_{\varphi}, v_{\theta}\right)$ : yaw, roll and pitch angular velocity from IMU.
- $v_{\delta}$ : tire angular velocity from steering potentiometer.

The actual angular velocities differ from the measured from the IMU and steering potentiometer. We model these differences by a zero-centered random variable with finite variance. If the vehicle state $X_{t-1}$ is in the form $X_{t-1}^{v}=\left(x_{t-1}, y_{t-1}, z_{t-1}, \psi_{t-1}, \varphi_{t-1}, \theta_{t-1}, \delta_{t-1}\right) \quad$ sampling algorithm for $X_{t}^{v} \approx p\left(X_{t}^{v} \mid u_{t}, X_{t-1}^{v}\right)$ s presented as the following;

$\hat{v}_{\delta}=v_{\delta}+\beta_{1} \cdot \operatorname{sample}\left(\sigma_{\delta}\right), \hat{\delta}_{t}=\delta_{t-1}+\Delta t . \hat{v}_{\delta}$
$\hat{v}_{\psi}=v_{\psi}+\beta_{2} \cdot \operatorname{sample}\left(\sigma_{\psi}\right), \hat{\psi}_{t}=\psi_{t-1}+\Delta t . \hat{v}_{\psi}$
$\hat{v}_{\varphi}=v_{\varphi}+\beta_{3} \cdot \operatorname{sample}\left(\sigma_{\varphi}\right), \hat{\varphi}_{t}=\varphi_{t-1}+\Delta t . \hat{v}_{\varphi}$
$\hat{v}_{\theta}=v_{\theta}+\beta_{4} \cdot \operatorname{sample}\left(\sigma_{\theta}\right), \hat{\theta}_{t}=\theta_{t-1}+\Delta t . \hat{v}_{\theta}$
$\hat{x}_{\theta}=x_{\text {odo }}+\beta_{5} \cdot \operatorname{sample}\left(\sigma_{o d o}\right)$
$\left[\begin{array}{c}\hat{x}_{t} \\ \hat{y}_{t} \\ \hat{z}_{t}\end{array}\right]=\left[\begin{array}{c}x_{t-1} \\ y_{t-1} \\ z_{t-1}\end{array}\right]+\left[C_{b}^{n}\left(\hat{\psi}_{t}, \hat{\varphi}_{t}, \hat{\theta}_{t}\right)\right]\left[\begin{array}{c}\hat{x}_{o d o} \operatorname{Cos}\left(\hat{\delta}_{t}\right) \\ 0 \\ 0\end{array}\right]$
Return $\left(\hat{x}_{t}, \hat{y}_{t}, \hat{z}_{t}, \hat{\psi}_{t}, \hat{\varphi}_{t}, \hat{\theta}_{t}, \hat{\delta}_{t}\right)$

where $\operatorname{sample}(\sigma)$ is normal or triangular distribution with zero mean and variance $\sigma$.

\section{Autonomous Navigation Architecture}

The autonomous navigation architecture has been depicted in the Fig 4. As shown in the architecture, we enhance a wide range of sensors for robust navigation and localization. As a reference global map for navigation, we use the Google Earth data to define the driving path for vehicle and define the positions of the landmarks. We use the onboard sensors including IMU/ odometer and steering angle to estimate the three dimensional vehicle dynamic model. As we use a low-cost GPS receiver which generally has enormous errors, we proposed a multi observations particle filter with joint observations model to compensate the localization errors. The path planning module generates suitable path according to the current positions, predefined path and obstacles in the local map. Once the intended path of the vehicle has been calculated by the path planner, the appropriate low level commands have been generated for vehicle actuators.

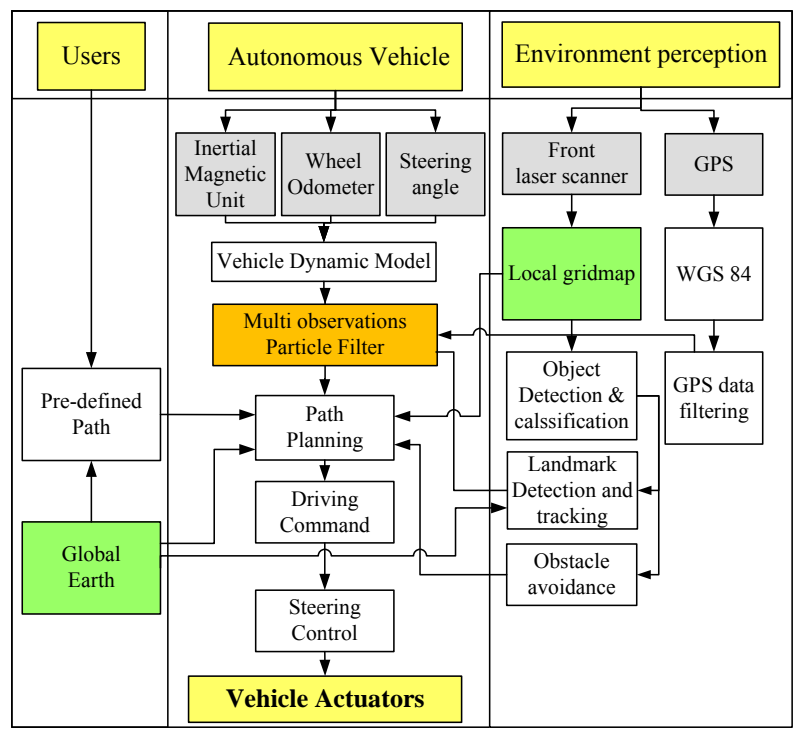

Fig. 4 Autonomous navigation and localization architecture 


\section{Experiments and Results}

\subsection{Vehicle platform}

We use an electric car which has been equipped and enhanced with an onboard array of sensors and computers as shown in Fig. 5. Our vehicle is Small Electric Vehicle (SEV) which has been enhanced for fully autonomous driving in both urban and nonurban environment.

\subsubsection{Sensors}

Four laser scanners have been installed on vehicle for obstacle avoidance, pedestrian detection and local navigation. The Inertial Magnetic Unit (IMU) with GPS provides data from 3 accelerometers, 3 gyroscopes, a 3D magnetic field sensor and GPS information. The IMU internal digital signal processor combines the embedded sensors to provide the filtered attitude of the vehicle (roll, pitch, heading to true north) and the position (latitude, longitude and altitude). We have installed three potentiometers to read position and direction of the accelerator, brake throttle and steering wheel. Moreover, a rotary encoder is installed in a left rear wheel of the vehicle for measuring the traveled distance and speed of the vehicle.

\subsubsection{Actuators}

A DC motor is installed on vehicle to control the steering wheel for autonomous driving. Control computer sends the steering wheel position voltage signal to the analog positional control and the positional control device generates suitable command to DC servo motor driver. Vehicle Electric Control Unit (ECU) controls the engine torque and position of accelerator throttle by information from accelerator sensor. The control computer controls the accelerator throttle by setting desire input voltage to the acceleration control of ECU. Finally, we utilize a compact ball screw linear motion actuator for autonomous control of vehicle brake throttle.

\subsubsection{Control unit}

A computer has been used to control the vehicle actuators and manage data from different sensors. We installed the Linux (Vine Linux 4.2) operating system for real time planning and control of vehicle. Control boards including AD/DA, DIO, Memory Link and motion control boards have been connected to the control computer.

\subsubsection{Data processing unit}

A powerful computer has been installed for processing the sensors data and mapping environment. The control computer gathers data from the different sensors and sends them to control computer The data processing computer reads the sensors data and processes them and sends back the processed information which are necessary for real time control to the control computer.

\subsection{Results}

We use $\mathrm{C}++$ and Mobile Robot Programming Toolkit (MRPT) for designing the navigation architecture software ${ }^{(14)}$. Different test courses have been designed including flat and up and down slopes to evaluate the efficiency of the localization algorithm.

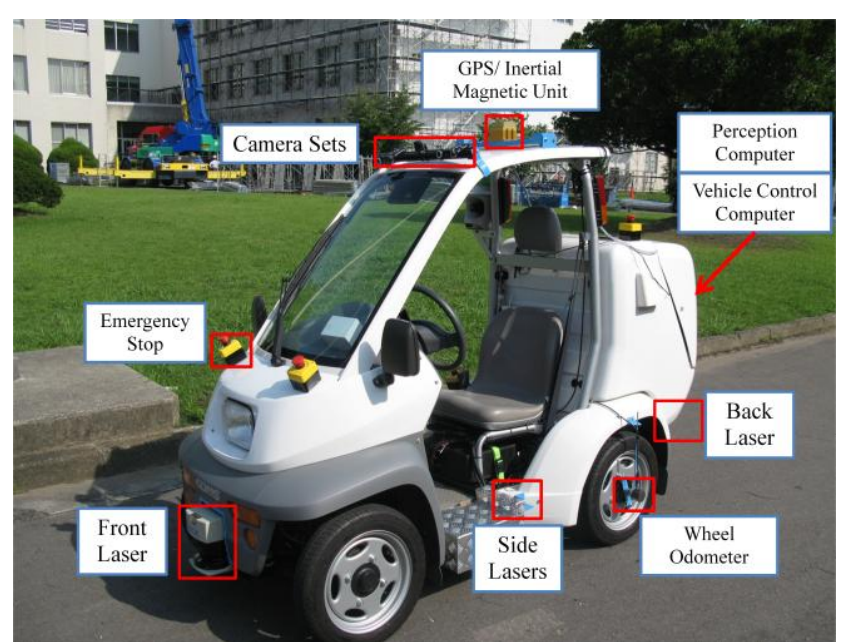

Fig. 5 Autonomous vehicle platform

The ground truth of the path has been recorded by RTK GPS with about $20 \mathrm{~mm}$ accuracy. We did some experiments inside our campus as we have limited for autonomous driving at outside. We have selected the driving path and the positions of the landmarks from Google Earth. We limit the number of samples to $M=20,000$ for particle filter. The results of three different courses have been shown in Fig 6. The blue lines shows the ground truth which have been recorded by accurate RTK GPS, the purple dots shows the quality of GPS receiver and the red lines shows the vehicle position according the vehicle prediction and sensors as shown in Fig.6(a). We put an initialization landmark for initializing the vehicle positions in start point of each experiments as shown in Fig. 6(a).

Fig. 6(a) depicts a rotary course about 220 meters including two landmarks. As you can see the Fig 6(a) the proposed localization method can compensate the GPS errors and follow

the path designed in the Google Earth. Fig 6(b) shows the 270 meters course in narrow road which starts from library and goes to the parking area. As we use low-cost IMU, the gyro drift causes enormous error in the localization of the vehicle in the Google Earth map. This error has been compensated by detecting the first landmark as shown in Fig 6(b). Fig 6(c) shows the experiments for about 210 meters including two up and down slopes. As shown in the experiments, the particle filter with joint observations is able to compensate the error of individual observations and generate near ground truth path. We did also some experiments to evaluate the robustness of the proposed localization and navigation algorithm, when intentionally some landmarks have not detected and missed in the experiments. The vehicle continues the autonomous driving by detecting the road curb and keeping safe lateral distance from left and right curb until it detects the next landmark.

\section{Conclusion}

We have proposed a practical low-cost localization and navigation architecture for urban autonomous driving. We use Google Earth maps as a reference global map for defining the driving path and landmark positions. To enhance the Google Earth data and improve the localization precision, we proposed an architecture to fuse different data from vehicle onboard sensors to 
estimate the vehicle state when individual observations such as GPS are noisy. The localization and navigation architecture can be extended to real autonomous driving in urban environment with low-cost sensors. In the future, we are trying to evaluate the localization and navigation method by gathering data from the real urban environment and do the simulation.

\section{References}

(1) A. Brown and Y. Lu, Performance test results of an integrated GPS/MEMS inertial navigation package," in Proc. ION GNSS Conf., Long Beach, CA, pp. 1251-1256 Mar. 2004.

(2) D. Obradovic, H. Lenz, and M. Schupfner, Fusion of map and sensor data in a modern car navigation system," J. VLSI Signal Process., vol. no. 1/2, pp. 111-122, Nov. 2006.

(3) J. Huang and H.-S. Tan, A low-order DGPS-based vehicle positioning system under urban environment," IEEE/ASME Trans. Mechatron., vol. 5, no. 1/2, pp. 567-575, Oct. 2006.

(4) J. Marias, M. Berbineau, and M. Heddebaut, tand mobile GNSS availability and multipath evaluation tool," IEEE Trans. Veh. Technol., vol. 54, no. 5, pp. 1697-1704, Sep. 2005.

(5) D. Lapucha, R. Barker, and H. Zwaan, Wide area carrier phase positioning," Eur. J. Navig., vol. 3, no. 1, pp. 10-16, Feb. 2005.

(6) K. Dixon, Satellite positioning systems: Efficiencies, performance and trends," Eur. J. Navig., vol. 3, no. 1, pp. 58-63, Feb. 2005.

(7) I. Skog and P. Händel, In-Car Positioning and Navigation Technologies-A Survey," IEEE Transactions on Intelligent Transportation Systems, vol. 10, no. 1, pp. 4-21, Mar. 2009.

(8) http://www.mapple.co.jp/.

(9) http://www.google.com/earth/index.

(10) E. Bekir, Introduction to Modern Navigation Systems, World Scientific Publishing, 2007.

(11) F. Caron, M. Davy, E. Duflos and P. Vanheeghe, "Particle filtering for multisensor data fusion with switching observation models: application to land vehicle positioning," IEEE Transactions on Signal Processing, vol. 55, no. 6, pp. 2703-2719, June 2007.

(12) H. Tehrani Niknejad, S. Mita and L. Han, Multi-Sensor Data Fusion for Autonomous Vehicle Navigation through Adaptive Particle Filter," in Proc. IEEE Intelligent Vehicle Symposium (IV)., Jun 2010, pp. 752-759.

(13) K. Klasing, D. Wollherr, and M. Buss, "A clustering method for efficient segmentation of 3d laser data," in Proceedings IEEE ICRA, 2008.

(14) http://babel.isa.uma.es/mrpt/index.php/Main_Page .

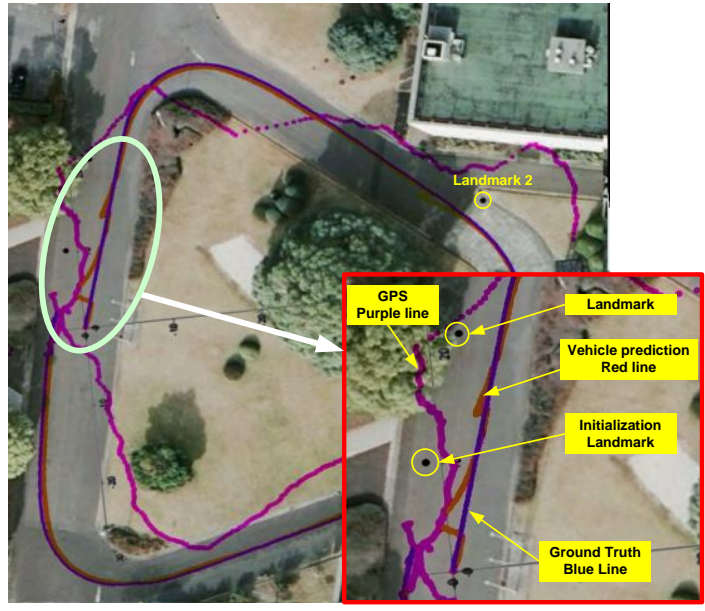

(a) Rotary course

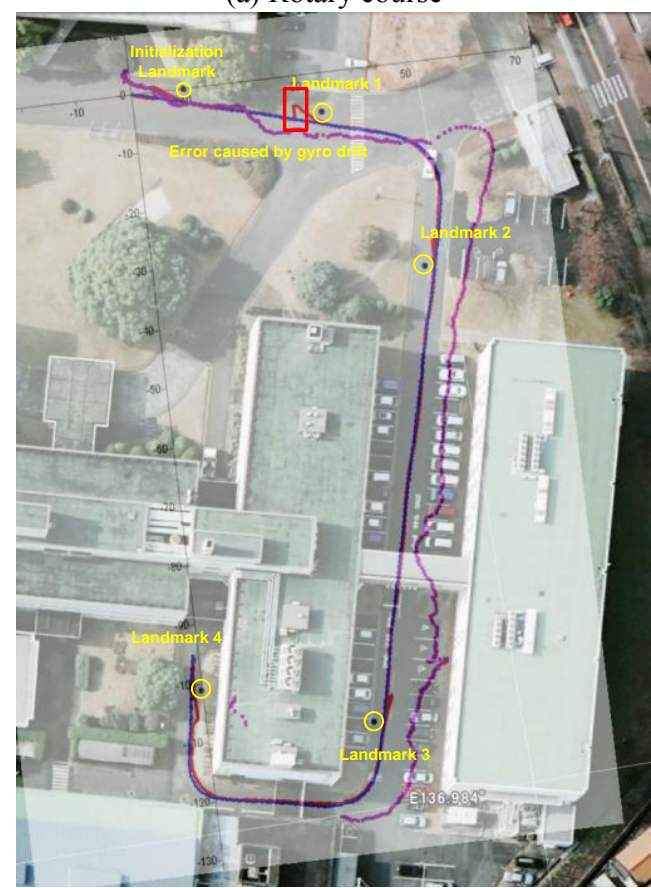

(b) Parking course

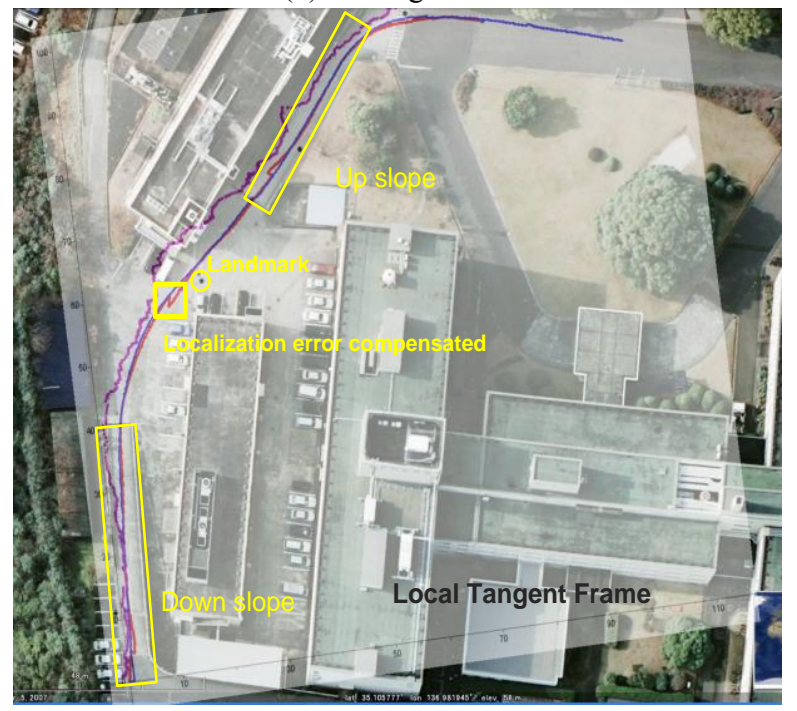

(c) Up and down slope course

Fig. 6 Autonomous navigation and control experiments 